\title{
Mechanistic Insights into Immunological Therapy for Targeting Diabetic Retinopathy
}

Mohammad Anas, Pallabi Banerjee, Nidhi Singh and Imteyaz Qamar*

School of Biotechnology, Gautam Buddha University, Greater Noida, U.P. -201312, INDIA.

*Corresponding author: Imteyaz Qamar, School of Biotechnology, Gautam Buddha University, Greater Noida, U.P. -201312, INDIA.

Received Date: April 26, 2021; Accepted Date: May 01, 2021; Published Date: May 11, 2021.

Citation: M Anas, P Banerjee, N Singh, I Qamar. (2021) Mechanistic Insights into Immunological Therapy for Targeting Diabetic Retinopathy. Journal of Endocrinology and Disorders. 5(3): Doi: 10.31579/2640-1045/073

Copyright: (C) 2021 Imteyaz Qamar. This is an open-access article distributed under the terms of the Creative Commons Attribution License, which permits unrestricted use, distribution, and reproduction in any medium, provided the original author and source are credited.

\begin{abstract}
Diabetic retinopathy (DR) is a common complication amongst patients that have diabetes. It is a leading cause of blindness in middle age people. A large proportion of patients who have diabetes develop retinopathy. There are several immunological reasons associated with the pathophysiology of this disease. Role of several mediators that increase the oxidative stress and have a pro-inflammatory effect which leads to capillary occlusion and neovascularization (NV). Increased vasopermeability due to disruption of the blood-retinal barrier (BRB) leading to diabetic macular edema (DME). Immunotherapies utilise different compounds and target various inflammatory molecules like TNF- $\alpha$ and pathways such as PPAR $\gamma$ for treatment of this progressive disease. Inflammatory and proinflammatory pathways are found to have an essential role in promoting DR; therefore, targeting them provides a useful technique for curing DR.
\end{abstract}

Keywords: diabetic retinopathy; anti-vascular endothelial growth factors; Neovascularization; diabetic macular edema; leukostasis, Inflammation; NSAIDs

\section{Introduction}

The current statistical data suggests that India would be regarded as diabetic capital in the near future. According to Kaveeshwar and Cornwall (2014), reported 31.7 million confirmed cases of diabetes mellitus (DM) in India in the year 2000. The mentioned figure is estimated to rise to 79.4 million by 2030, the largest number in any country in the world. Almost all types of diabetics, either Type $1 \mathrm{DM}$ or Type $2 \mathrm{DM}$, are expected to develop diabetic retinopathy (DR) in the long run [1]. Diabetes constitutes a group of metabolic disorders that share a common characteristic feature of hyperglycemia, i.e., elevated glucose level in the blood. This could be of two types: type $1 \mathrm{DM}$ where insulin is deficient or type $2 \mathrm{DM}$ characterized by variable degrees of insulin resistance in the cells, impaired insulin secretion from islet cells of the pancreas and/or glucose over-production. If this hyperglycemic state is not controlled, the chronic exposure can give rise to various complications - nephropathy, neuropathy and retinopathy [2]. Amongst the various ocular complications caused by DM, Diabetic retinopathy is the most commonly observed preventable cause of blindness. Other ocular complications from DM include glaucoma, cataract and ocular surface diseases.

Diabetic retinopathy (DR) is microangiopathy, i.e. pathology in small retinal vessels like arterioles, venules and capillaries. Features of DR include retinal capillary occlusion and leakage, breaking the blood-retinal barrier; hence, exudate formation due to leakage of leaked lipoproteins. This is followed by neovascularization [3]. In the initial days of Diabetic retinopathy, patients are mostly asymptomatic. Symptoms might start precipitating as the microangiopathy progresses. In such cases, symptoms observed are like blurred vision, floaters and flashes, distorted vision, and in later stages, there could be partial or even total loss of vision.

The most common reason for the loss of vision in patients with DR is diabetic macular edema (DME). DME is characterized by swelling or thickening of the macula due accumulation of fluid in the macula because of the breakdown of the blood-retinal barrier [4]. DME can occur at any DR stage, which can lead to distortion of visual images and a reduction in visual acuity [5].

Presently, the treatment plans for diabetic retinopathy aim at managing the microvascular complications by intravitreal pharmacologic agents, laser photocoagulation and vitreous surgery.

Anti-VEGF (vascular endothelial growth factors) agents are the most used intravitreal agent nowadays to manage both early and advanced stages of DR. Although conventional laser therapy provides stabilization of visual acuity, anti-VEGF therapy can help in developing visual improvement with less ocular adverse effects [6]. Here, immunological therapy is discussed for targeting diabetic retinopathy.

\section{Pathophysiology}

A number of factors are involved in the progression of diabetic retinopathy. These factors can be genetic, epigenetic, or environmental, which makes the development of DR quite complex. The main contributing factors in pathogenesis are elevated blood sugar levels (Hyperglycemia), hyperlipidemia, hypertension, neuronal dysfunction and inflammation [7]. Dysfunctioning of different retinal cells such as glial, muller, ganglion, endothelial cells, etc. are also engaged in this 
process [6]. The dysfunctioning of blood microcapillaries in the retina can lead to an increase in its membrane permeability followed by its obliteration. The breakdown of the blood-retinal barrier (BRB) will lead to macular edema and formation of abnormal new blood vessels (Neovascularization). The major factor responsible for structural and functional changes associated with DR is the elevated level of glucose concentration in the blood. Hyperglycemia causes advanced glycation end products (AGEs) formation, protein kinase $\mathrm{C}$ activation, increase in oxidative stress and promotes the polyol pathway [8]. AGEs and its receptor have a pro-inflammatory effect and increase the neurodegenerative process [9]. AGEs also play a part in the protein kinase $\mathrm{C}$ activation that is responsible for processes such as occlusions in the capillary, increase in oxidative stress, production of pro-inflammatory proteins and elevated vasopermeability [10]. Hyperglycemia activates reactive oxygen species (ROS) that damage the retinal cells, further stimulating cellular damage [11].

\section{Inflammation in DR:}

Inflammation and many pro-inflammatory compounds like vascular endothelial growth factor, different cytokines, chemokines, angiotensin II, eicosanoid, etc. have an additive effect in the pathogenesis of diabetic retinopathy [12]. Microvascular inflammation leads to diabetic macular edema (DME), blockage in blood supply to tissues leading to hypoxic condition (ischemia), and formation of abnormal new blood vessels [13]. Recent studies infer that VEGF is affiliated to ICAM-1 upregulation and is able to induce conformational changes in tight junctions of the endothelial cells which may result in endothelial cell disruption and leukostasis as VEGF is the robust vasoactive factor that induces angiogenesis and increase the vascular permeability [14]. Sustained hyperglycemia and hypoxia can induce VEGF upregulation because of vasoconstriction and capillary loss. Retinal cells like Muller cells, ganglions, endothelial cells, and pericytes are highly active in response and metabolism; therefore, they may have a part in producing VEGF as reported by some studies [15]. DR patients have been observed with high levels of TNF- $\alpha$, which is a pro-inflammatory cytokine in the ocular fibrovascular membranes. Also, in the vitreous samples from the DR patients, upregulated levels of chemokines have been observed such as CCL5, CCL2, CXCL12, CXCL8 and CXCL10. Studies suggest that the increased levels of the inflammatory molecules such as TNF- $\alpha$, IL-1 $\beta$, CXCL12 and CCL5 contribute to the pathogenesis of DR [16].

\section{Macular edema and vascular leakage:}

Diabetic macular edema (DME) or simply macular edema is a result of blood-retinal barrier disruption which consists of inner barrier and the outer barrier. The impairment of the BRB is carried out by several vasoactive factors. Increased vasopermeability due to distortion of tight junctional complexes between endothelial cells in the retina and vascular leakage is promoted by VEGF, resulting in extracellular accumulation [17]. BRB breakdown is boosted by PKC, which further increases the vasopermeability and encourages VEGF activation. The responsibility to maintain the inner BRB and provide vascular stability is carried out by pericytes. Dysfunctioning or loss of pericytes will evidently disturb the integrity and working of the inner $\mathrm{BRB}$, which will lead to microaneurysm formation, increased vasopermeability and capillary dilatation [18].

\section{Retinal ischemia and neovascularization:}

The occlusion in blood capillaries stimulates retinal nonperfusion, which leads to neovascularization. In diabetic patients, increased levels of VEGF play a key role in vascular obstruction leading to ischemia through complex mechanisms. This level of VEGF can be reduced subsequently by pan-retinal photocoagulation. When leukocytes adhere to retinal vessels, it can cause endothelial cell death, vascular blockage and loss of pericyte. This pro-angiogenic mechanism is known as retinal vessel leukostasis. Neovascularization in the retina may also be intensified by a pro-angiogenic factor that is a platelet-derived growth factor (PDGF) [13].

\section{Immunotherapies}

Immunotherapy infers to a kind of therapy or treatment that is utilised for stimulating the immune system of a person for treating a certain disease. DR is a chronic sight-threatening disease and current treatment measures adopted for its remedy stay ineffective to an extent. Current strategies employ the use of treatments like laser photocoagulation, pan-retinal photocoagulation, intravitreal injections, intravitreal anti-VEGF, etc. Due to the lack of efficacy of these treatment methods, scientists are carrying out research to understand the immunological insights of this disease and to take advantage of this knowledge which can help in designing a robust strategy for the immunotherapy of DR.

\section{Intravitreal Corticosteroids:}

For the treatment of DME in DR intravitreal corticosteroids are being used where there is a negligible response to anti-VEGF therapy [19]. Corticosteroids are potent anti-inflammatory agents that can target large spectra of mediators that are involved in the progression of DME like chemokines, leukostasis, VEGF, TNF- $\alpha$, and phosphorylation of tightjunction proteins. The treatment of DME includes dexamethasone intravitreal implant, fluocinolone acetonide intravitreal implant and off label triamcinolone acetonide [6].

\section{Non-Steroid Anti-Inflammatory Drugs (NSAIDs):}

NSAIDs have an anti-inflammatory effect and inhibit the formation of eicosanoid mediated by cyclooxygenase (COX) enzyme [20]. The NSAIDs target the COX enzymes. Mainly the COX-2 enzyme is active at the sites of inflammation and stimulates the production of proinflammatory factors such as PGE2 $\alpha$ and PGF2. The inhibition of this enzyme downregulates the production of PGE2 and further reduces the inflammatory reactions induced due to diabetes. Aspirin and salicylatebased drugs, i.e., NSAIDs had a beneficial effect in reducing the incidence of diabetic retinopathy as reported in clinical studies [6].

\section{Anti-Inflammatory Pathways:}

Diabetes induces the upregulation of the pro-inflammatory pathways to exaggerate the retinal inflammation. This retinal inflammation is further enhanced by the impairment of endogenous anti-inflammatory mechanisms like the proliferator-activated receptor (PPAR) $\gamma$ pathway. Impaired PPAR $\gamma$ is a sign of DR in DR patients, as observed in scientific studies [21]. During Inflammation, inflammatory cytokines induce oxidative stress and stimulate the expression of other molecules involved in inflammation. Therefore, inhibition of the inflammatory cytokines results in downregulating the oxidative stress and pathogenesis of DR in experimental models [22].

\section{Blocking of RAS:}

The renin-angiotensin system is involved in diabetic as well as hypertension-induced retinal inflammation. The RAS pathway has crosstalk with many other pathways of AGEs and oxidative stress. Animal models induced with diabetes had shown to prevent vascular damage, oxidative stress and inflammation when the specific blockade of the RAS was performed [23].

\section{Blocking the Inflammatory molecules:}

TNF- $\alpha$ is a key molecule for inflammatory reactions as it induces the formation of ROS, activates NF- $\kappa$ B pathway and upregulates the adhesion molecules expression like ICAM1 on the endothelial surface, which results in the recruitment of leukocytes. Therefore, TNF- $\alpha$ was targeted to study the anti-TNF- $\alpha$ therapy in the model animals induced with 
diabetes and some clinical cases. TNF- $\alpha$ trap (a soluble TNF- $\alpha$ receptor/Fc construct, Etanercept) was subcutaneously administered in diabetic rats and it significantly blocked the retinal inflammation, reduced the retinal cell injury and vascular leakage [24]. In clinical trials, a TNF$\alpha$ neutralizing antibody was used, which improved visual acuity and reduced macular thickness in the patients [25]. Anti-CD49a neutralizing antibody targets the VLA-4 via blocking leukocyte recruitment which reduces levels of TNF- $\alpha$, VEGF, vessel leakage and leukostasis [26].

\section{Anti-Inflammasome therapy:}

Inflammasomes are innate immune system receptors that have a significant role in inflammation. The inflammasomes include a central protein, an adaptor protein called apoptosis speck-like protein (ASC) and caspase- 1 protein. Caspase -1 induces maturation of cytokines like IL- $\beta$ and IL-8 that causes inflammation. The inflammasomes such as NLRP1, NLRP3, NLRC4 and AIM2 have a role in pathogenesis in various disorders including diabetic retinopathy and glaucoma [27]. The compound methylene blue has been identified to be effective in controlling diabetic retinopathy by inhibiting inflammasome NLRP3 [28]. Vitamin D3 (Cholecalciferol) dampens the activity of NLRP3 by the reduction of ROS and aids in intervention for DR [29]. Type 1 Interferons can inhibit the NLRP3 inflammasomes by reducing the IL-1 $\beta$ which inhibits NLRP1b via IL-10R and STAT3 signaling [27].

\section{Discussion}

DR is retinopathy occurred in the retina due to diabetes. Diabetes is a group of metabolic disorders with a general phenotype of uncontrolled and unhealthy high blood glucose levels or hyperglycemia. Chronic hyperglycemia can lead to the pathogenesis of DR. It is fundamentally microangiopathy in small retinal vessels leading to vascular endothelial dysfunction [30].

Many metabolic pathways are adversely affected due to hyperglycemia and lead to retinopathy.

1. Increased polyol pathway: When glucose becomes excessive in the body system, it is reduced to sorbitol by the activation of the polyol pathway. Normally, this sorbitol is converted to fructose by the action of dehydrogenase enzyme in order to cross cell membranes. But due to the high glucose concentration, production of sorbitol also becomes higher, and this sorbitol gets accumulated because of enzymatic limitation. This accumulated sorbitol causes osmotic damage to retinal cells [31].

2. Protein kinase $\mathrm{C}(\mathrm{PKC})$ pathway activity: This pathway is triggered by an increased amount of DAG or Di-acyl glycerol. This activates PKC, which further enhances permeability and upregulation of vascular endothelial growth factors (VEGF) in the retina [32]. These two things affect retinal hemodynamics and abnormal neovascularization.

It is also responsible for leukostasis (abnormal clumping of leukocytes) causes capillary occlusion [33].

3. VEGF itself is an important parameter that leads to major microvascular complications of DM. Normally, it is produced by Retinal epithelial cells, pericytes and Mueller cells of the retina. The production is increased in hypoxic condition as a compensatory mechanism causing neovascularization. This increased intravitreal VEGF is also a factor responsible for DME, i.e. Diabetic macular edema [34].

4. Chronic oxidative damage or high amount of reactive oxygen species also facilitate microvascular complications.

These whole sequences of events also cause the release of inflammatory mediators in the retina, and patients usually suffer from chronic inflammation [35].
Patients may be apparently asymptomatic in initial stages but later may complain of blurred vision, floaters and flashes, dark areas in vision, impaired colour vision and in advanced disease, there could be partial to a total loss of vision.

In recent times, DR is classified into two categories - 1. non-proliferative diabetic retinopathy (NPDR) or 2. Proliferative diabetic retinopathy (PDR). These two categories are provided by Early treatment diabetic retinopathy study (ETDRS) classification. It is based on clinical findings like microaneurysms, intraretinal haemorrhages, venous abnormalities such as beading, intraretinal microvascular abnormalities (IRMA), and neovascularization (NV) [3].

\section{NPDR:}

- In Mild (NPDR), There are a few microaneurysms; most of these patients are reviewed annually. The review may vary from 6-12 months, depending upon the severity of signs and systemic factors.

- Moderate NPDR: less than 20 microaneurysms. Hard yellow exudates, cotton wool spots, and venous beading may also be present, but these findings are confined to the only quadrant. Every six months of review is recommended in this case.

- Severe NPDR: It is identified as any of following clinic features; Microaneurysms in all four quadrants; Venous beading in two or more quadrants; IRMA in one or more quadrants. The review is done every four months.

-Very severe NPDR: This category includes two or more of the criteria for severe NPDR. Every 2-3 months review is required.

\section{PDR}

- It is mostly based on neovascularization of the disc (NVD) and neovascularization elsewhere (NVE) [3].

- Due to ischemia (caused by leukocytic occlusion, inflammation and above discussed factors), NV is seen to develop and grow at the optic nerve (NVD) and elsewhere in the retina except the optic disc (NVE). In other words, NV develops at the border zone of the perfused and nonperfused retina [36].

- There are three categories of PDR provided by ETDRS classification.

- mild to moderate: If NVD is present in less than one-third disc area and NVE is in less than half-disc area. Here, treatment should be planned according to the severity of signs, the patient's systemic factors and personal circumstances. If it is left untreated, every two months review is advised.

- High risk: if NVD is in more than 1/3 area or Any NVD with vitreous or pre-retinal haemorrhage or NVE $>1 / 2$-disc area with vitreous or preretinal haemorrhage. Such patients require treatments as per the surgeon's/ophthalmologist's evaluation and surgical plan. Possible treatment courses include laser photocoagulation, intravitreal Anti VEGF or/and triamcinolone, pars plana vitrectomy and lipid-lowering drugs.

- Advanced diabetic eye disease: features of this category are Preretinal (retro-hyaloid) and/or integral haemorrhage, Tractional retinal detachment, Tractional retinoschisis, Rubeosis iridis (iris neovascularization). In these patients, 'Pars plana vitrectomy' is recommended [37].

\section{Conclusion}

Diabetic retinopathy is a chronic progressive vision-threatening disease of retinal blood microcapillary caused due to prolonged hyperglycemia \& other conditions linked to diabetes, like hypertension. As per the data available, $33 \%$ of patients living with diabetes are diagnosed with signs 
of diabetic retinopathy. Once the diagnosis is made, a suitable treatment plan is chosen. Current treatment strategies in DR include use of Antiangiogenic therapy like anti-VEGF (mostly used). There are some other anti-angiogenic agents which are under trials and research like Squalamine + Ranibizumab therapy and Nesvacumab. Anti-inflammatory therapy by intravitreal administration of steroid and/or Non-steroidal antiinflammatory drugs (NSAIDs).

Inflammatory and pro-inflammatory pathways have a significant role in the pathophysiology of the disease involving aneurysm, microangiopathy, neovascularization and neural death. Enhancing endogenous antiinflammatory pathways and devising strategies for blocking the proinflammatory pathways are promising strategies for the effective management of DR. Anti-inflammatory compounds such as Anti-CD49a neutralizing antibody and Vitamin $\mathrm{C} \& \mathrm{E}$ are effective interventions for the management of DR. Anti-inflammasome compounds such as methylene blue, and vitamin D can also be used in the management of DR.

\section{Abbreviations Used}

AGEs- Advanced Glycation End products

BRB- Blood Retinal Barrier

COX-Cyclooxygenase

DM- Diabetes Melitus

DME- Diabetic Macular Edema

DR- Diabetic Retinopathy

ETDRS- Early Treatment Diabetic Retinopathy Study

ICAM1- Intercellular Adhesion Molecule 1

IRMA- Intraretinal Microvascular Abnormalities

MMP- Matrix Metallopeptidase

NPDR- Non-Proliferative Diabetic Retinopathy

NSAID- Non-Steroidal Anti-Inflammatory Drug

NV- Neovascularization

NVD- Neovascularization of the Disc

NVE- Neovascularization Elsewhere

PDGF- Platelet-derived Growth Factor

PDR- Proliferative Diabetic Retinopathy

PKC- Protein Kinase C

RAS- Renin-Angiotensin System

ROS- Reactive Oxygen Species

TNF $\alpha$ - Tumor Necrosis Factor $\alpha$

VEGF- Vasoactive Endothelial Growth Factor

VLA- Very Late Antigen

\section{Acknowledgements}

We thank our authors from Gautam Buddha University who provided insight and expertise that greatly assisted the study. This review was not supported by any grant.

\section{Conflicts Of Interest}

The author declares no conflicts of interest.

\section{References}

1. Kaveeshwar, S. A., \& Cornwall, J. (2014). The current state of diabetes mellitus in India. The Australasian Medical Journal, 7(1), 45-48.

2. Kawahito, S., Kitahata, H., \& Oshita, S. (2009). Problems associated with glucose toxicity: role of hyperglycemia-induced oxidative stress. World Journal of Gastroenterology, 15(33), 41374142.

3. Fong, D. S., Aiello, L., Gardner, T. W., King, G. L., Blankenship, G., Cavallerano, J. D., \& Klein, R. (2004). Retinopathy in diabetes. Diabetes care, 27(suppl 1), s84-s87.

4. Romero-Aroca, P., Baget-Bernaldiz, M., Pareja-Rios, A., LopezGalvez, M., Navarro-Gil, R., \& Verges, R. (2016). Diabetic Macular Edema Pathophysiology: Vasogenic versus Inflammatory. Journal of diabetes research, 2156273.

5. Gonzalez, V. H., Campbell, J., Holekamp, N. M., Kiss, S., Loewenstein, A., Augustin, A. J., Ma, J., Ho, A. C., Patel, V., Whitcup, S. M., \& Dugel, P. U. (2016). Early and Long-Term Responses to Anti-Vascular Endothelial Growth Factor Therapy in Diabetic Macular Edema: Analysis of Protocol I Data. American journal of ophthalmology, 172, 72-79.

6. Wang, W., \& Lo, A. (2018). Diabetic Retinopathy: Pathophysiology and Treatments. International journal of molecular sciences, 19(6), 1816.

7. Bhagat N, Grigorian RA, Tutela A, Zarbin MA (2009) Diabetic macular edema: pathogenesis and treatment. Surv Ophthalmol 54:1-32.

8. Ghanem AA, Elewa A, Arafa LF (2011) Pentosidine and Ncarboxymethyl-lysine: biomarkers for type 2 diabetic retinopathy. Eur J Ophtahlmol 21:48-54.

9. Tarr, J. M., Kaul, K., Chopra, M., Kohner, E. M., \& Chibber, R. (2013). Pathophysiology of diabetic retinopathy. ISRN ophthalmology. 343560.

10. Coral K, Angayarkanni N, Gomathy N, et al. (2009) Homocysteine levels in the vitreous of proliferative diabetic retinopathy and rhegmatogenous retinal detachment: its modulating role on lysyl oxidase. Invest Ophthalmol Vis Sci 50:3607-3612.

11. Madsen-Bouterse SA, Kowluru RA (2008) Oxidative stress and diabetic retinopathy: pathophysiological mechanisms and treatment perspectives. Rev Endocr Metab Disord 9:315-327

12. Rübsam, A., Parikh, S., \& Fort, P. E. (2018). Role of Inflammation in Diabetic Retinopathy. International journal of molecular sciences, 19(4), 942.

13. Adamis, A. P., \& Berman, A. J. (2008). Immunological mechanisms in the pathogenesis of diabetic retinopathy. Seminars in immunopathology, 30(2), 65-84.

14. Antonetti DA, Barber AJ, Hollinger LA et al (1999) Vascular endothelial growth factor induces rapid phosphorylation of tight junction proteins occludin and zonula occluden 1. J Biol Chem 274:23463-23467

15. D. S. Aiello, L.; Gardner, T. W.; King, G. L.; Blankenship, G.; Cavallerano, J. D.; Ferris, F. L.; Klein, R. (2004). Retinopathy in Diabetes. Diabetes Care, 27(Supplement 1), S84-S87.

16. Zhang, W., Liu, H., Rojas, M., Caldwell, R. W., \& Caldwell, R. B. (2011). Anti-inflammatory therapy for diabetic retinopathy. Immunotherapy, 3(5), 609-628.

17. Wang J, Xu X, Elliott MH et al (2010) Müller cell-derived VEGF is essential for diabetes-induced retinal inflammation and vascular leakage. Diabetes 59:2297-2305

18. Bandello, F., Lattanzio, R., Zucchiatti, I., \& Del Turco, C. (2013). Pathophysiology and treatment of diabetic retinopathy. Acta diabetologica, 50(1), 1-20.

19. Lattanzio R., Cicinelli M.V., Bandello F. (2017) Intravitreal steroids in diabetic macular edema. Dev. Ophthalmol.;60:78-90. 
20. Kim SJ, Flach AJ, Jampol LM. (2010) Nonsteroidal antiinflammatory drugs in ophthalmology. Surv Ophthalmol.;55(2):108-133.

21. Tawfik A, Sanders T, Kahook K, Akeel S, Elmarakby A, AlShabrawey M. (2009) Suppression of retinal peroxisome proliferator-activated receptor gamma in experimental diabetes and oxygen-induced retinopathy: role of NADPH oxidase. Invest Ophthalmol Vis Sci.;50(2):878-884.

22. Rojas M, Zhang W, Lee DL, et al. (2010) Role of IL-6 in angiotensin II-induced retinal vascular inflammation. Invest Ophthalmol Vis Sci. 51(3):1709-1718.

23. Zhang JZ, Xi X, Gao L, Kern TS. (2007) Captopril inhibits capillary degeneration in the early stages of diabetic retinopathy. Curr Eye Res. 32(10):883-889.

24. Joussen AM, Poulaki V, Mitsiades N, et al. (2002) Nonsteroidal anti-inflammatory drugs prevent early diabetic retinopathy via TNF-alpha suppression. Faseb J. 16(3):438-440.

25. Sfikakis PP, Markomichelakis N, Theodossiadis GP, Grigoropoulos V, Katsilambros N, Theodossiadis PG. (2005) Regression of sight-threatening macular edema in type 2 diabetes following treatment with the anti-tumor necrosis factor monoclonal antibody infliximab. Diabetes Care. 28(2):445-447.

26. Iliaki E, Poulaki V, Mitsiades N, Mitsiades CS, Miller JW, Gragoudas ES. (2009) Role of alpha 4 integrin (CD49d) in the pathogenesis of diabetic retinopathy. Invest Ophthalmol Vis Sci. 50(10):4898-4904.

27. Yerramothu, P., Vijay, A. K., \& Willcox, M. (2018). Inflammasomes, the eye and anti-inflammasome therapy. Eye (London, England), 32(3), 491-505.
28. Hao, J., Zhang, H., Yu, J., Chen, X., \& Yang, L. (2018). Methylene blue attenuates diabetic retinopathy by inhibiting NLRP3 inflammasome activation in STZ-induced Diabetic Rats. Ocular Immunology and Inflammation, 27(5), 836-843.

29. Raman, K. S, \& Matsubara, J. A. (2020). Dysregulation of the NLRP3 inflammasome in diabetic retinopathy and potential therapeutic targets. Ocular Immunology and Inflammation.

30. Mathebula, S. D. (2015). Polyol pathway: A possible mechanism of diabetes complications in the eye. African vision and eye health, 74(1), 5.

31. Lorenzi, M. (2007). The polyol pathway as a mechanism for diabetic retinopathy: attractive, elusive, and resilient. Experimental diabetes research.

32. Aiello LP, Avery RL, Arrigg PG et al (1994) Vascular endothelial growth factor in ocular fluids of patients with diabetic retinopathy and other retinal disorders. N Engl J Med 331:1480-1487.

33. Frank, R. N. (2002). Potential new medical therapies for diabetic retinopathy: protein kinase $\mathrm{C}$ inhibitors.

34. Gupta, N., Mansoor, S., Sharma, A., Sapkal, A., Sheth, J., Falatoonzadeh, P, \& Kenney, M. C. (2013). Diabetic retinopathy and VEGF. The open ophthalmology journal, 7, 4.

35. Kowluru, R. A., \& Chan, P. S. (2007). Oxidative stress and diabetic retinopathy. Experimental diabetes research.

36. Bresnick, G. H., De Venecia, G., Myers, F. L., Harris, J. A., \& Davis, M. D. (1975). Retinal ischemia in diabetic retinopathy. Archives of ophthalmology, 93(12), 1300-1310.

37. Danis, R. P., \& Davis, M. D. (2008). Proliferative diabetic retinopathy. In Diabetic retinopathy (pp. 29-65). Humana Press.
This work is licensed under Creative Commons Attribution 4.0 License

\section{To Submit Your Article Click Here: Submit Manuscript}

DOI: $10.31579 / 2692-9759 / 073$

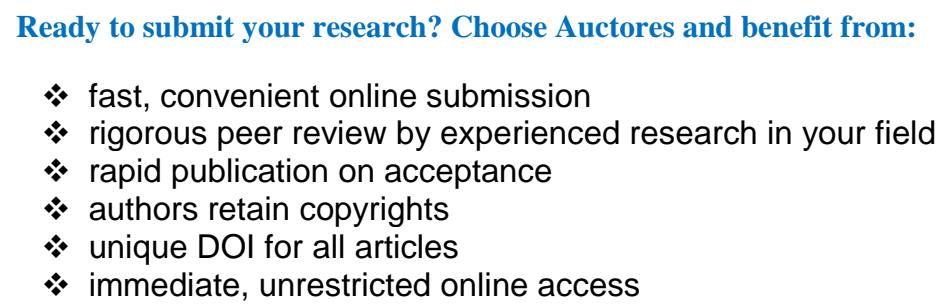

At Auctores, research is always in progress.

Learn more www.auctoresonline.org/journals/endocrinology-anddisorders- 